\title{
Novel immune genes associated with excessive inflammatory and antiviral responses to rhinovirus in COPD
}

Katherine J Baines ${ }^{1,2^{*}+}$, Alan C-Y Hsu ${ }^{1,2^{*}+}$, Melinda Tooze ${ }^{1,2}$, Lakshitha P Gunawardhana ${ }^{1,2}$, Peter G Gibson ${ }^{1,2,3}$ and Peter $A B$ Wark ${ }^{1,2,3}$

\begin{abstract}
Background: Rhinovirus (RV) is a major cause of chronic obstructive pulmonary disease (COPD) exacerbations, and primarily infects bronchial epithelial cells. Immune responses from BECS to RV infection are critical in limiting viral replication, and remain unclear in COPD. The objective of this study is to investigate innate immune responses to RV infection in COPD primary BECs (pBECs) in comparison to healthy controls.

Methods: Primary bronchial epithelial cells (pBECs) from subjects with COPD and healthy controls were infected with RV-1B. Cells and cell supernatant were collected and analysed using gene expression microarray, qPCR, ELISA, flow cytometry and titration assay for viral replication.

Results: COPD pBECs responded to RV-1B infection with an increased expression of antiviral and pro-inflammatory genes compared to healthy pBECs, including cytokines, chemokines, RNA helicases, and interferons (IFNs). Similar levels of viral replication were observed in both disease groups; however COPD pBECs were highly susceptible to apoptosis. COPD pBECs differed at baseline in the expression of 9 genes, including calgranulins S100A8/A9, and 22 genes after RV-1B infection including the signalling proteins pellino-1 and interleukin-1 receptor associated kinase 2. In COPD, IFN- $\beta / \lambda 1$ pre-treatment did not change MDA-5/RIG-I and IFN- $\beta$ expression, but resulted in higher levels IFN- $\lambda 1, C X C L-10$ and CCL-5. This led to reduced viral replication, but did not increase pro-inflammatory cytokines.

Conclusions: COPD pBECs elicit an exaggerated pro-inflammatory and antiviral response to RV-1B infection, without changing viral replication. IFN pre-treatment reduced viral replication. This study identified novel genes and pathways involved in potentiating the inflammatory response to RV in COPD.
\end{abstract}

Keywords: COPD, Immune response, Viral infection, Gene expression

\section{Background}

Chronic obstructive pulmonary disease (COPD) is responsible for an increasing burden of illness and death around the world. COPD is chronic airway disease, characterized by incompletely reversible airflow obstruction, and symptoms of cough and sputum production. These symptoms can be worsened with exposure to microbial

\footnotetext{
*Correspondence: katherine.baines@newcastle.edu.au; alan.hsu@newcastle. edu.au

${ }^{\dagger}$ Equal contributors

'Priority Research Centre for Asthma and Respiratory Diseases, The University of Newcastle, Callaghan, NSW, Australia

${ }^{2}$ Virus, Infections/Immunity, Vaccines, \& Asthma, Hunter Medical Research Institute, Lot 1, Kookaburra Circuit, New Lambton Heights, NSW, Australia Full list of author information is available at the end of the article
}

infections [1]. Rhinoviruses (RVs) are the most frequently detected viruses during acute exacerbation [2], and infection is associated with rapid decline in lung function and severe symptoms that often requires hospitalization [2]. However the specific mechanism that leads to this enhanced susceptibility and severe symptoms following RV infection is not well understood.

Bronchial epithelial cells (BECs) are the primary site of $\mathrm{RV}$ infection, where the infection occurs both in the upper and lower respiratory epithelium equally [3]. RV can infect other cells including airway macrophages, however the virus does not replicate in these cells [4], which demonstrates the important role of BECs in the first line of defence against invading pathogens. As RVs
C Biomed Central

(c) 2013 Baines et al.; licensee BioMed Central Ltd. This is an Open Access article distributed under the terms of the Creative Commons Attribution License (http://creativecommons.org/licenses/by/2.0), which permits unrestricted use, distribution, and reproduction in any medium, provided the original work is properly cited. 
are endocytosed into BECs viral RNAs are preferentially recognized by the RNA helicase melanoma differentiation-associated gene -5 (MDA-5, also known as IFIH1), which then signals for the induction of type I interferon (IFN- $\alpha / \beta$ ) and type III IFNs (IFN- $\lambda 1 / 2 / 3$ ). These IFNs then signal for the expression of over 300 IFN-stimulated genes (ISGs) including IFNs, CCL-5, CXCL-10, and the RNA helicases MDA-5 and retinoic acid-inducible gene-I (RIG-I or DDX58) [5]. These ISGs amplify IFN responses and induce apoptosis and limit viral replication. CCL-5 and CXCL-10 recruit cytotoxic $\mathrm{T}$ cells to kill virus-infected cells [5].

RV RNAs are also recognized by toll-like receptor (TLR) -3 and via nuclear factor - kappa B (NF-kB) signalling triggering the release of pro-inflammatory cytokines such as IL-6, leading to acute inflammation and fever [6]. Subjects with COPD are known to have increased inflammatory cytokines that drive increased recruitment of neutrophils due to heightened chemoattractant CXCL-8, and the neutrophils then phagocytose replicating viruses and limit viral spread [7]. However the mechanisms underlying the severe outcomes to infection in COPD and the role of BECs responses to virus infection remains unclear. Here we hypothesized that primary BECs (pBECs) from COPD subjects have an exaggerated inflammatory response and abnormal antiviral response to RV infection compared to healthy control pBECs. In this study, pBECs from subjects with COPD and healthy controls were infected $\mathrm{RV}-1 \mathrm{~B}$, and the immune responses to infection were assessed using whole genome microarray analysis, allowing the opportunity for discovery of novel regulators of abnormal responses in COPD.

\section{Methods}

\section{Rhinovirus}

RV-1B was obtained from the American Tissue Culture Collection (ATCC, USA). Virus was propagated using RD-ICAM-1 cells, clarified by centrifugation and viral titres were determined using 50\% Tissue Culture Infective Dose $\left(\mathrm{TCID}_{50}\right)$.

\section{Subject recruitment}

Subjects with COPD were recruited; defined by a previous smoking history and fixed airflow limitation on spirometry with an $\mathrm{FEV}_{1} / \mathrm{FVC}$ ratio $<70 \%$, and $\mathrm{FEV}_{1}<80 \%$ predicted and classified by the GOLD criteria. Those with GOLD Stage III (severe COPD) FEV $130-50 \%$ predicted, and Stage IV (very severe COPD) $\mathrm{FEV}_{1}<30 \%$ were included. COPD subjects were ex-smokers (at least one year abstinent) and did not use inhaled corticosteroids for two weeks prior to bronchoscopy. Healthy non-smoking controls with no evidence of airflow obstruction, bronchial hyper-responsiveness to hypertonic saline challenge, or chronic respiratory symptoms were recruited. A clinical history, examination and spirometry were performed. Subjects were excluded if they had symptoms of an acute respiratory tract infection a month prior. All subjects gave written informed consent. The study was approved by The University of Newcastle Human Research Ethics.

\section{Cell culture and viral infection}

Human pBECs were obtained by endobronchial brushing during fibre-optic bronchoscopy in accordance with standard guidelines, and cultured as described previously [8]. Cells were seeded at $1 \times 10^{5}$ and cultured in BEBM basal media with supplements (BEGM, Lonza). At passage 2 when the cells reached $80 \%$ confluency in 24-well plates, virus infection was performed at a multiplicity of infection (MOI) of 20 for 1 hour with shaking. After 1 hour incubation viral inoculum was removed and fresh BEBM basal media supplemented with $0.5 \%$ BSA was then added. For IFN pre-treatment experiments, human recombinant IFN- $\beta$ (10U) and IFN- $\lambda 1$ protein $(1 \mathrm{ng} / \mathrm{mL}$; $R \& D$ Systems) was used to pre-treat pBECs for $24 \mathrm{hrs}$ before RV-1B infection in the designated experiments. For all experiments samples were collected at $6 \mathrm{hrs}$ for whole genome gene expression microarray and real-time PCR (qPCR) and 24hrs for ELISA/CBA and viability/ apoptosis.

\section{Whole genome gene expression microarray}

pBEC RNA was extracted, 500ng amplified and 750ng cRNA hybridised to Illumina's HumanRef- 8 V3 BeadChips as previously described [9].

\section{Real-time PCR (qPCR)}

RNA was extracted, reverse transcribed to cDNA and real-time PCR was performed as previously described [9]. Briefly, RNA (200ng) was reverse-transcribed to cDNA using the high capacity cDNA reverse transcription kit (Applied Biosystems, Foster City,USA). Taqman qPCR primer and probes for the target genes were purchased in kit form (Applied Biosystems, Foster City, USA). PCR primers and probes were combined with Taqman gene expression master mix as per manufacturer's instructions in duplicate singleplex real-time PCRs (7500 Real Time PCR System, Applied Biosystems). Fold change results were calculated using $2^{-\Delta \Delta C t}$ relative to the internal reference gene (18S) and the mean of all samples.

\section{ELISA}

IFN- $\lambda 1 / 3$, CXCL-10, IL-6, TNF- $\alpha$ and CCL-5 concentration was assessed using ELISA kit (IFN- $\lambda 1 / 3$, R\&D Systems, lower detection limit of $31.25 \mathrm{pg} / \mathrm{mL}$ ) and cytometric bead array (CXCL-10, IL-6, TNF- $\alpha$ and CCL-5, BD 
Biosciences, lower detection limit of $10 \mathrm{pg} / \mathrm{mL}$ ) according to the manufacturer's instructions.

\section{Viability/Apoptosis}

Apoptosis was measured using Annexin V-PE Apoptosis Detection Kit I (Becton Dickinson) according to manufacturer's instructions [10]. Briefly, infected and noninfected cells were stained with Annexin V-PE and 7-AAD analysed by flow cytometry. Annexin V-PE positive and 7-AAD negative were determined to be apoptotic, Annexin V-PE and 7-AAD positive were necrotic and Annexin V-PE and 7-AAD negative were live cells.

\section{Viral replication}

Viral replication was determined using both TCID $_{50}$ and relative RNA copy number using a standard curve created using serial dilution of RV-1B RNA.

\section{Data analysis}

Data analysis preformed using Stata 9 or GraphPad Prism 5 and reported as mean with standard error of means (SEM) for normally distributed data and median (quartile [Q] 1, Q3) for nonparametric data. Statistical comparisons were performed by using multiple comparisons ANOVA for parametric data and the Kruskal-Wallis test for nonparametric data. $\mathrm{P}<0.05$ was considered significant.

\section{Whole genome gene expression microarray analysis}

Microarray data were exported by using Genome Studio (Illumina) and analyzed by using GeneSpring GX11 (Agilent Technologies). Data were log-transformed, normalized, and baseline-converted to the median of all samples. Data were filtered, and only genes flagged as present $(<0.05$ detection $\mathrm{P}$ value $)$ in all samples were included in the further analysis. Hierarchical clustering analysis was performed by using the Pearson centered algorithm with complete linkage. The Pearson algorithms cluster samples on the basis of their correlation coefficients, whereas complete linkage measures that the distance between 2 clusters is the greatest distance between the members of the 2 clusters. Differential gene expression was determined by using ANOVA with Tukey post hoc testing ( $\mathrm{P}<0.05$ adjusted for multiple comparisons by using the Benjamini-Hochberg method) and fold change $>2$.

\section{Results}

Gene expression profiles of healthy control and COPD $\mathrm{pBECs}$ at baseline and in response to RV-1B infection

Primary BECs from ten subjects with moderate and severe COPD and ten healthy controls were assessed in vitro for their immune response to RV-1B infection. Patient characteristics and details of optimisation of experimental conditions are summarised in Additional file
1: Table $\mathrm{S} 1$ of the online supplement. Gene expression at baseline of the non-infected media controls was first examined and showed a different expression profile between COPD and healthy pBECs (Table 1). There were 9 genes that were highly up-regulated in COPD pBECs, including genes involved in innate immunity, cell repair, and some with protease/anti-protease activity.

Infection of pBECs with RV-1B led to changes in the gene expression profile from baseline, and differences between healthy and COPD $\mathrm{pBEC}$ responses were assessed. RV-1B infection caused significant up-regulation of 23 entities corresponding to 20 genes in healthy control pBECs (Figure 1A), and 48 entities corresponding to 42 genes were significantly induced in COPD pBECs (Figure 1B). Of the genes that were induced in both COPD and healthy control pBECs, all were up-regulated to higher levels in COPD compared to that in healthy pBECs (Figure 1C). There was also an additional 22 genes upregulated in COPD pBECs in response to RV-1B infection (Table 2).

\section{RV-1B infection of COPD pBECs induced higher inflammatory and antiviral responses compared to healthy controls}

Microarray analysis showed that COPD pBECs induced an overall higher level of inflammatory and antiviral gene expression after RV-1B infection (Additional file 1: Table S2). The increase of selected genes detected by microarray were further validated by $\mathrm{qPCR}$ and confirmed by ELISA at the protein level.

RV-1B infection in healthy pBECs did not cause an up-regulation of the inflammatory genes IL-6 and TNF- $\alpha$, however RV-1B infection led to a significant upregulation of IL-6 (Figure 2A), but not TNF- $\alpha$ (Figure 2B) from media control in COPD pBECs. RNA helicases MDA-5 (Figure 2C) and RIG-I (Figure 2D) were up-regulated after RV-1B infection of both healthy and COPD pBECs, however the induction was significantly higher in the COPD group. Induction of IFN- $\lambda 1$, and ISGs CCL-5 and CXCL-10 was significantly higher in COPD pBECs after infection compared to that in healthy pBECs (Figure $2 \mathrm{~F}-\mathrm{H}$ ). IFN- $\beta$ was only induced in COPD pBECs after infection (Figure 2E).

All proteins were induced above media control and no difference was observed at this baseline level between healthy and COPD pBECs (data not shown). Both healthy and COPD pBECs induced significant levels of IL-6 and TNF- $\alpha$ at $24 \mathrm{hr}$ after RV-1B infection (Figure 3A - B), with IL- 6 being significantly higher in COPD pBECs. Similarly IFN- $\lambda 1$, CCL-5, and CXCL-10 protein was also significantly higher in COPD than in healthy pBECs after RV-1B infection (Figure 3C - E).

Surprisingly the high inflammatory and antiviral induction in COPD pBECs did not affect viral replication 
Table 1 Differential gene expression between baseline COPD pBECs and healthy control pBECs

\begin{tabular}{llll}
\hline SYMBOL & GENE NAME & FOLD CHANGE & \\
\hline Protease and Antiproteases & & & P value \\
\hline MMP10 & Matrix metallopeptidase 10 & 2.94 & 0.0029 \\
PI3 & Peptidase inhibitor 3 (Elafin) & 2.22 & 0.0287 \\
ADAM19 & ADAM metallopeptidase domain 19 & 2.13 & 0.0090 \\
Innate immunity & & & 0.0189 \\
\hline S100A8 & S100 calcium binding protein A8 & 2.63 & 0.0143 \\
S100A9 & S100 calcium binding protein A9 & 2.32 & \\
Epithelial cell related processes & & & \\
\hline KRT6C & Keratin 6C & 10.01 & 0.0002 \\
RHCG & Rh family, C glycoprotein & 2.51 & 0.0033 \\
CNFN & Cornifelin & 2.23 & 0.0213 \\
SPRR2E & Small proline-rich protein 2E & 2.15 & 0.0017 \\
\hline
\end{tabular}

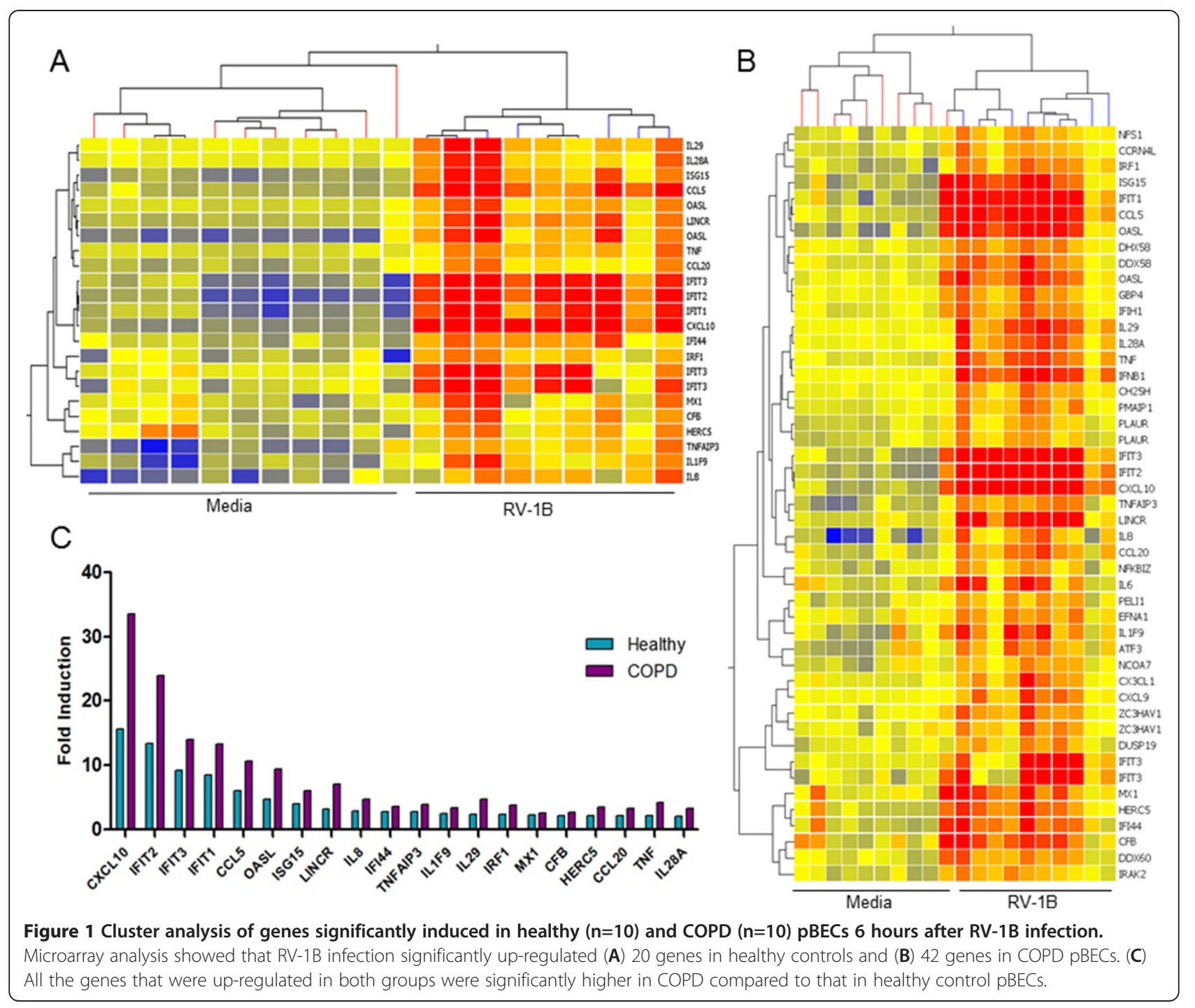




\begin{tabular}{|c|c|c|c|c|}
\hline SYMBOL & GENE NAME & FOLD CHANGE & $P$ value & PROBE ID \\
\hline IFNB1 & Interferon, beta 1 & 5.8 & $<0.0001$ & 1510669 \\
\hline IL6 & Interleukin 6 & 3.3 & 0.0022 & 4040576 \\
\hline RIG-I & Retinoic acid-inducible gene - I & 3.1 & $<0.0001$ & 4760703 \\
\hline ATF3 & Activating transcription factor 3 & 2.7 & 0.0007 & 4780128 \\
\hline CX3CL1 & Chemokine (C-X3-C motif) ligand 1 & 2.5 & $<0.0001$ & 630521 \\
\hline CXCL9 & Chemokine (C-X-C motif) ligand 9 & 2.5 & $<0.0001$ & 5570278 \\
\hline MDA-5 & Melanoma differentiation-associated gene -5 & 2.5 & $<0.0001$ & 4570441 \\
\hline \multirow[t]{2}{*}{ ZC3HAV1 } & Zinc finger $\mathrm{CCCH}$-type, antiviral 1 & 2.4 & $<0.0001$ & 37102434540382 \\
\hline & & 2.2 & $<0.0001$ & \\
\hline PMAIP1 & Phorbol-12-myristate-13-acetate-induced protein 1 & 2.4 & $<0.0001$ & 2750367 \\
\hline DHX58 & DEXH (Asp-Glu-X-His) box polypeptide 58 & 2.3 & $<0.0001$ & 3930681 \\
\hline NFS1 & NFS1 nitrogen fixation 1 homolog & 2.2 & $<0.0001$ & 7570411 \\
\hline $\mathrm{CH} 25 \mathrm{H}$ & Cholesterol 25-hydroxylase & 2.2 & $<0.0001$ & 1770593 \\
\hline CCRN4L & CCR4 carbon catabolite repression 4-like & 2.2 & $<0.0001$ & 460484 \\
\hline IRAK2 & Interleukin-1 receptor-associated kinase 2 & 2.2 & $<0.0001$ & 3930750 \\
\hline PELI1 & Pellino homolog 1 & 2.2 & 0.0007 & 3450092 \\
\hline NCOA7 & Nuclear receptor coactivator 7 & 2.1 & 0.0001 & 630091 \\
\hline \multirow[t]{2}{*}{ PLAUR } & Plasminogen activator, urokinase receptor & 2.1 & 0.0054 & 7305286220671 \\
\hline & & 2.1 & 0.0028 & \\
\hline DDX60 & DEAD (Asp-Glu-Ala-Asp) box polypeptide 60 & 2.1 & 0.0001 & 7610053 \\
\hline NFKBIZ & Nuclear factor of kappa light polypeptide gene enhancer in B-cells inhibitor, zeta & 2.1 & 0.0011 & 2470348 \\
\hline GBP4 & Guanylate binding protein 4 & 2.1 & $<0.0001$ & 1980524 \\
\hline EFNA1 & Ephrin-A1 & 2.1 & $<0.0001$ & 3390187 \\
\hline DUSP19 & Dual specificity phosphatase 19 & 2.0 & 0.0019 & 5260195 \\
\hline
\end{tabular}

(Additional file 1: Figure S1 of the online supplement), as viral RNA and viral titre at $24 \mathrm{hr}$ was similar in healthy and COPD pBECs. RV-1B infection in both healthy and COPD pBECs resulted in a significant decrease in cell viability (Additional file 1: Figure S2A of the online supplement), however there was more apoptosis in the RV infected COPD pBECs (Additional file 1: Figure S2B and E2C of the online supplement).

The heightened inflammatory response in COPD pBECs is due to enhanced immune signalling caused by RV-1B infection

In order to understand the mechanisms underlying the increased immune responses in COPD pBECs, we further examined the genes that are involved in immune signalling and that were up-regulated greater than 2 fold by $\mathrm{RV}-1 \mathrm{~B}$ infection in COPD but not healthy pBECs (Table 2). These genes were reviewed for their role in innate immunity in published literature (Pubmed), and those with known functions were mapped into the currently known intracellular innate immune signalling pathways. Figure 4 illustrates the key novel innate immune signalling genes involved in RV induced inflammatory responses in COPD pBECs. Signalling via IL-1 and TLR pathways was apparent, involving Pellino 1 (PELI1), IL-1 receptor-associated kinase-like 2 (IRAK2) and cholesterol 25-hydroxylase $(\mathrm{CH} 25 \mathrm{H})$. Oxidative stress appears important relating to the up-regulation of activating transcription factor 3 (ATF3) and guanylate binding protein 4 (GBP4), and phorbol-12-myristate-13acetate-induced protein 1 (PMAIP1 or Noxa) plays a role in apoptosis (Figure 4).

\section{Pre-treatment with IFN- $\beta$ and IFN- $\lambda 1$ led to enhanced} antiviral responses to RV-1B infection in both healthy and COPD pBECS

COPD pBECs produced a greater antiviral response to $\mathrm{RV}$ infection, but this was not associated with reduced viral replication. We sought to determine if IFN- $\beta / \lambda 1$ pre-treatment affects the inflammatory and antiviral responses to RV-1B infection. pBECs were primed with a combination of exogenous IFN- $\beta$ and IFN- $\lambda 1$ before infection. IFN $-\beta / \lambda 1$ pre-treatment led to a significant induction of IL- 6 and TNF- $\alpha$ mRNA in healthy control 


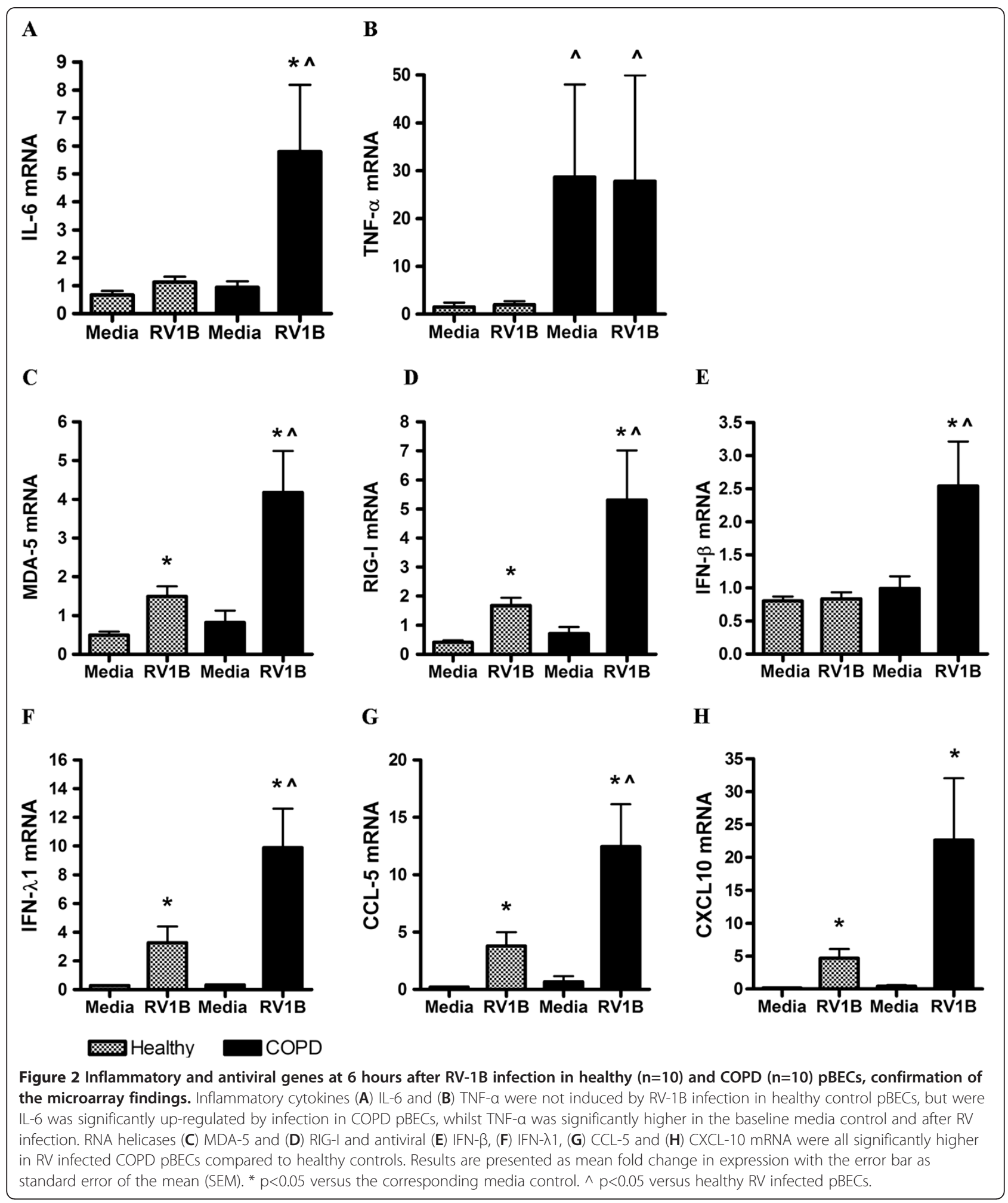

but had no effect in COPD pBECs (Figure 5A - B). While the RNA helicases and IFN- $\beta$ mRNA was significantly up-regulated in healthy control pBECs by the pretreatment, these genes were not induced in COPD $\mathrm{pBECs}$ following pre-treatment (Figure $5 \mathrm{C}-\mathrm{E}$ ). IFN- $\lambda 1$,
CCL-5, and CXCL-10 mRNA however were significantly induced in both pBECs groups after IFNs pre-treatment (Figure 5F - H). Surprisingly release of IL-6 and TNF- $\alpha$ protein did not increase with the up-regulated gene expression. IL- 6 protein was in fact reduced and TNF- $\alpha$ 

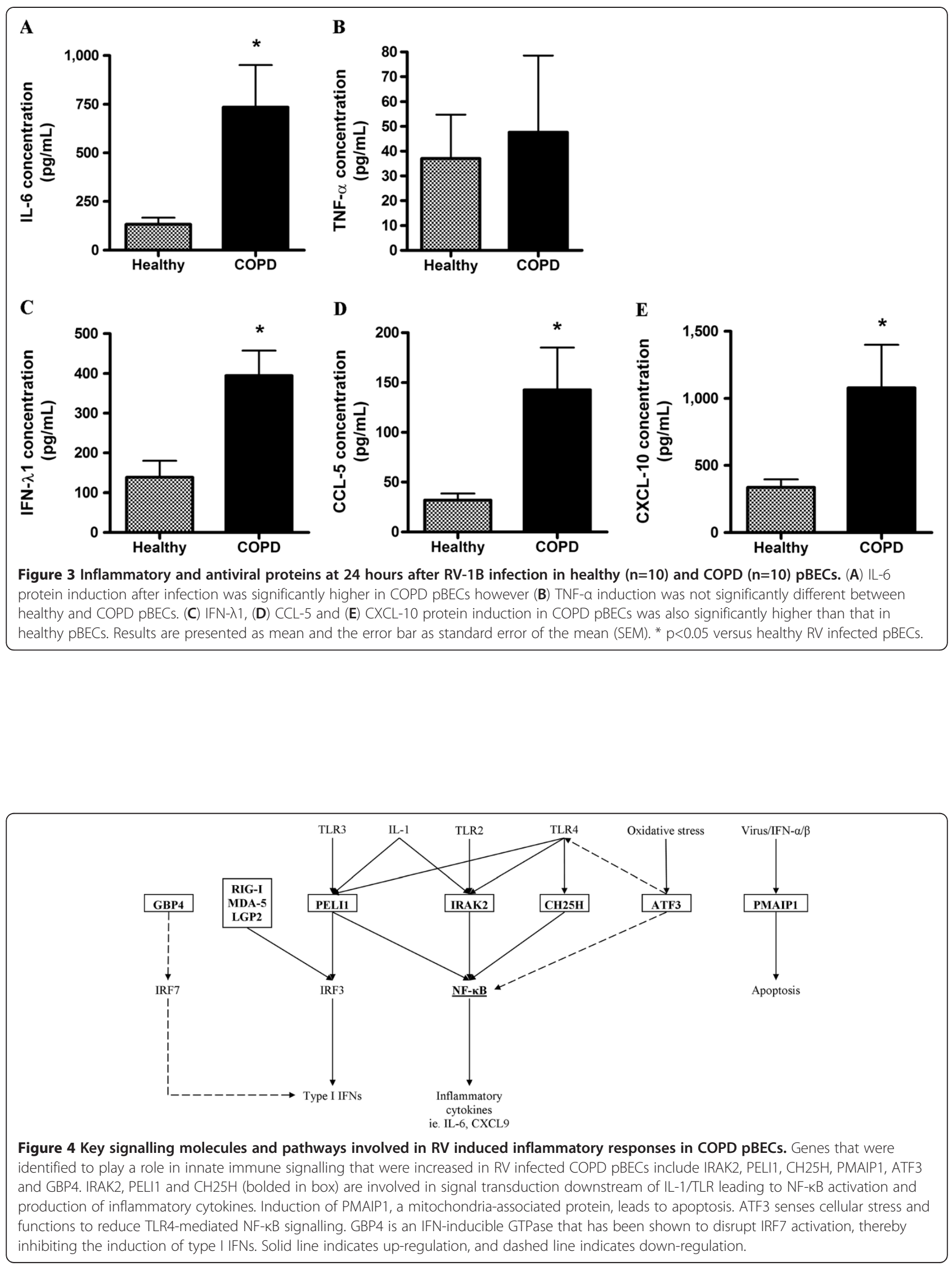


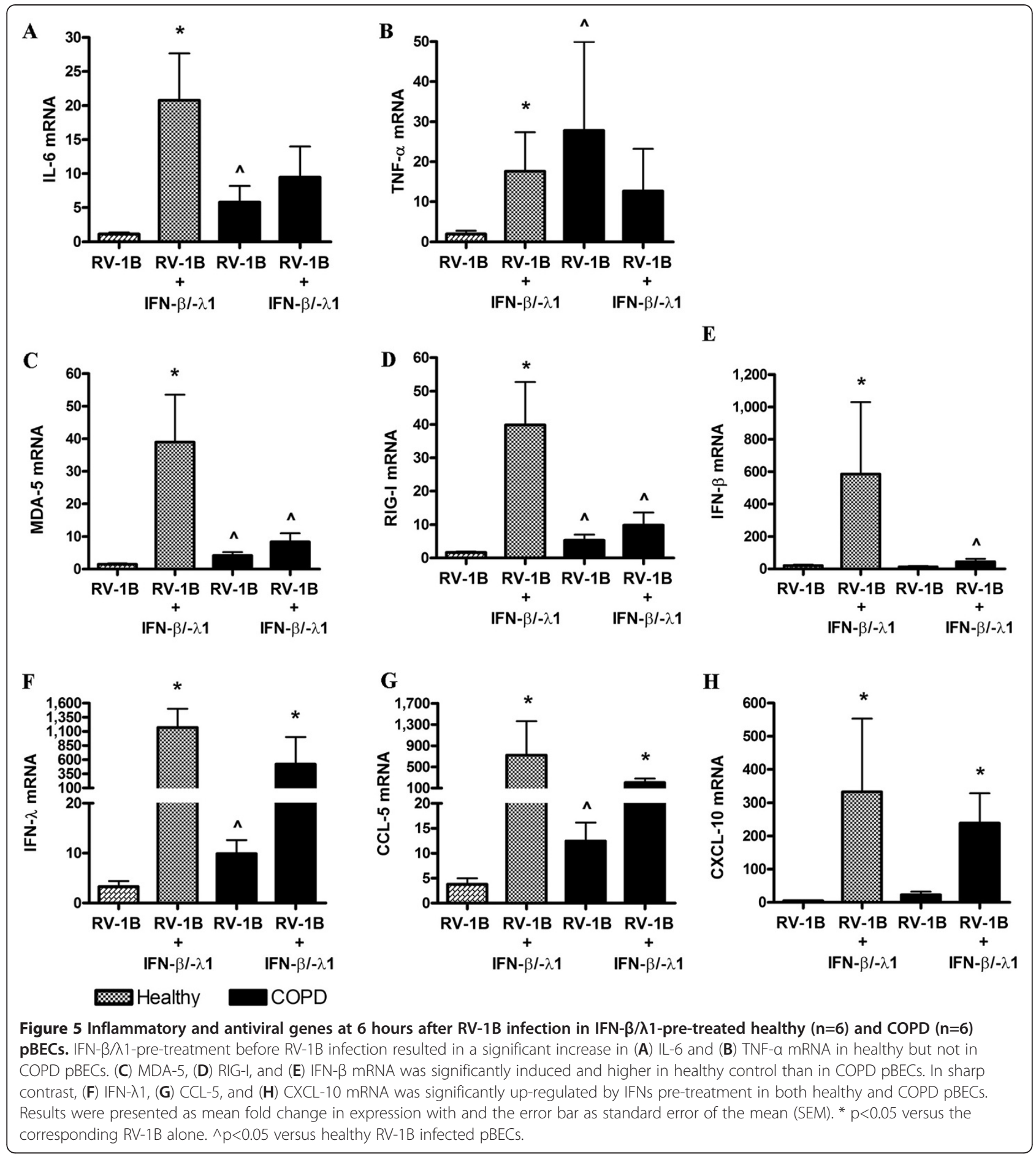

remained unaffected following IFN- $\beta / \lambda 1$ pre-treatment (Figure 6A - B). However, IFN- $\lambda 1$, CCL-5, and CXCL10 proteins were significantly induced in both healthy and COPD pBECs after pre-treatment (Figure 6C - E). The heightened antiviral responses also correlated with significantly reduced viral titre with exogenous IFN- $\beta / \lambda 1$ treatment (Additional file 1: Figure S3 of the online supplement).

\section{Discussion}

People with COPD are more susceptible to viral infection and suffer severe complications with worsened symptoms and frequent exacerbations following infection. This study investigated the transcriptional response of pBECs to RV infection and how this is altered in COPD. We show that the immune response of healthy and COPD cells was characterised by a robust up- 


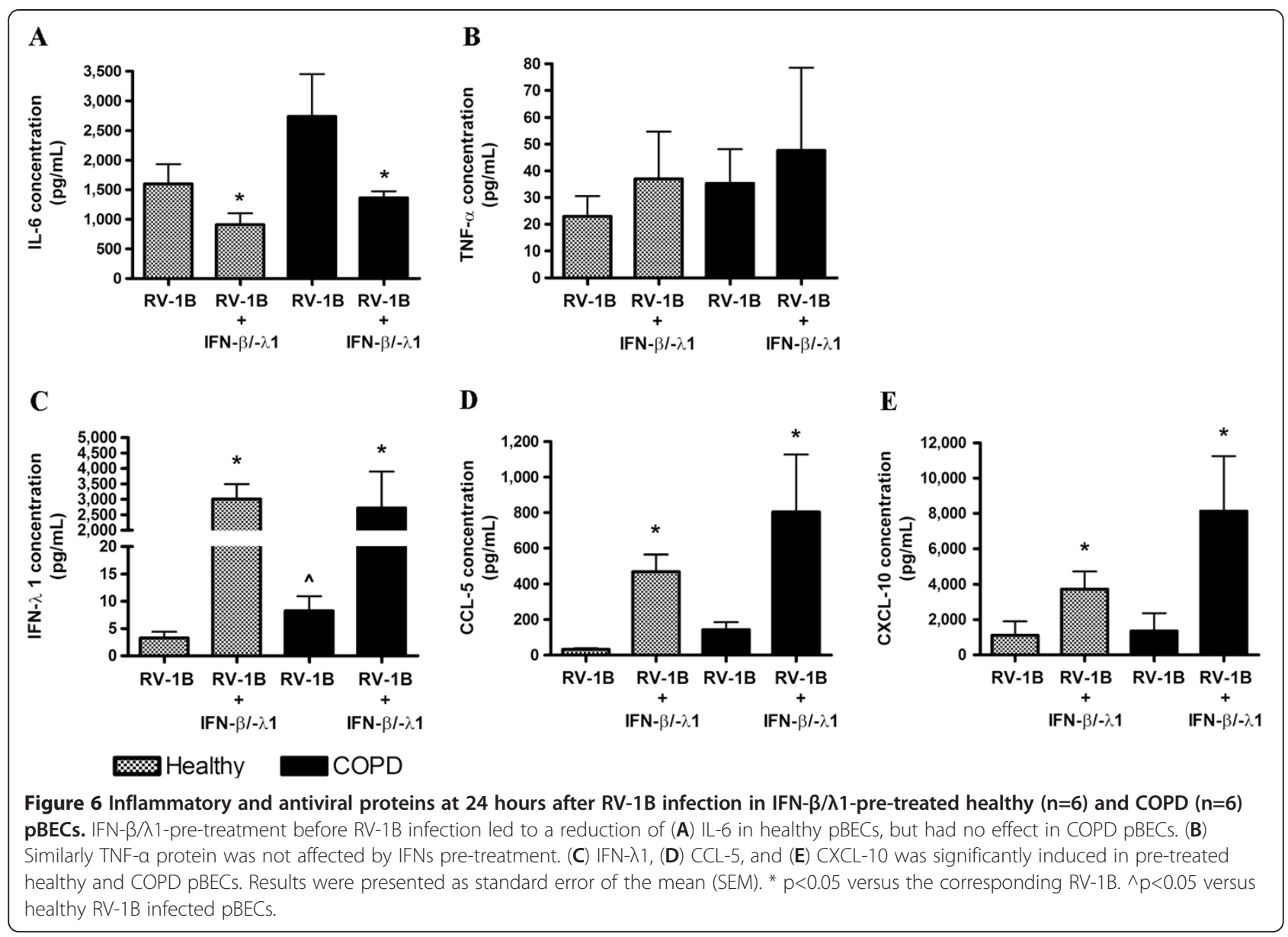

regulation of pro-inflammatory and antiviral pathways. However there were clear differences between the COPD and healthy pBECs, including up-regulation of inflammatory genes at baseline and dramatically exaggerated responses to RV infection. We identified 9 genes associated with COPD at baseline and 22 genes altered in COPD but not healthy $\mathrm{pBEC}$ in response to RV, not previously reported in COPD, but likely to be important in regulating the exaggerated virus induced inflammation. The increased gene expression in RV infected COPD pBECs also correlated with the corresponding protein release, and enhanced apoptosis; however this enhanced immune response did not reduce viral titre. Furthermore, IFN- $\beta / \lambda 1$ pre-treatment resulted in enhanced responses to $\mathrm{RV}-1 \mathrm{~B}$ in COPD and healthy pBECs, however COPD pBECs were unresponsive in terms of MDA-5/RIG-I and IFN- $\beta$ gene induction. Despite this abnormality IFN- $\beta / \lambda 1$ pre-treatment still led to significantly reduced viral titre.

The airway epithelium in COPD is exposed chronically to enhanced airway inflammation with increased numbers of neutrophils and lymphocytes that correlate with more severe airflow obstruction, despite the fact that the majority of subjects have ceased smoking, indicating that the airway inflammatory response in COPD becomes self-perpetuating $[11,12]$. We found several novel associations with 9 up-regulated genes in COPD pBECs, including the calgranulins S100A8 and S100A9, and proteases ADAM19 and MMP10. S100A8/A9 are small calcium-binding proteins with pro-inflammatory activity and have been reported to increase with steroid resistant neutrophilic inflammation [13]. ADAM19 and MMP10 are involved in tissue repair and remodelling, and single nucleotide polymorphisms in the gene locus containing ADAM19 has been associated with COPD [14]. These observations are consistent with other studies suggesting that the airways of COPD subjects undergo constant cellular repair due to the damages caused by cigarette smoke exposure [1]. Interestingly MMP10 and ADAM19 gene expression has also been shown to be upregulated in pBECs from subjects with asthma [15].

Upon RV-1B infection, both pBEC groups mounted a robust inflammatory response; however the response was more exaggerated in COPD pBECs. This was in accordance with other studies that showed a more vigorous inflammatory response in COPD pBECs against RV infection [16]. We have identified a number of novel 
genes whose expression were strongly up-regulated in COPD pBECs in response to RV-1B but not in healthy pBECs. These include important signalling molecules downstream of IL-1 and TLR2, 3 and 4 pathways such as PELI1 [17], IRAK2 [18] and $\mathrm{CH} 25 \mathrm{H}$ [19]. We have previously shown that PELI1 and IRAK2 are upregulated in the sputum of subjects with neutrophilic asthma [9]. This suggests that IRAK2 and PELI1 play a role promoting neutrophilic airway inflammation, which is triggered by RV infection of the epithelium in COPD. Recently, PELI1 has been shown to be important in regulating the innate immune response of the epithelium to RV, including CXCL-8 production and neutrophil recruitment, without interfering with IFN responses and viral replication [20]. Mouse models have shown that IRAK2 is critical in sustaining late phase inflammatory responses after TLR stimulation, leading to increased production of inflammatory cytokines [21]. These molecules may also be important in $\mathrm{T}$ cell related functions, such as T cell tolerance [22], and promotion of Th17 cell development [23].

Other important signalling proteins induced by RV in COPD pBECs include PMAIP1, ATF3 and GBP4. PMAIP1, a mitochondria-associated protein, promotes apoptosis [24]. ATF3 senses oxidative stress [25] and functions to reduce TLR4-mediated NF- $\mathrm{KB}$ signalling, therefore serving as negative feedback [26]. GBP4 is an IFN-inducible GTPase that has been shown to disrupt IRF7 activation, thereby inhibiting the induction of type I IFNs [27]. Also potentially important is the upregulation of Chemokine (C-X3-C motif) ligand 1 (CX3CL1), more commonly known as fractalkine, by RV-1B in COPD pBECs. Soluble CX3CL1 has a chemoattractant activity for $\mathrm{T}$ cells and monocytes; whereas membrane bound protein promotes adhesion of these leukocytes [28].

Antiviral responses such as IFN- $\beta$ and $-\lambda 1$ are critical in limiting viral replication, and were significantly higher in COPD pBECs. This was associated with the higher level of apoptosis after infection, but surprisingly did not affect viral titre in COPD, which was similar to the healthy group. This was in contrast to the study by Schneider et al. that showed enhanced antiviral responses associated with increased replication of RV-39 after infection in COPD [29]. Other studies have examined transcriptional responses to in vivo RV infection of healthy controls using nasal epithelial scrapings [30], or in vitro RV infection of healthy pBECs [31]. There are many similarities between the changes in gene expression found in reported studies compared to the current study, which includes upregulation of ISGs, antiviral genes, chemokines, and cytokines. However there are also differences in the healthy $\mathrm{pBEC}$ responses, including some genes previously reported to be upregulated by RV (e.g. IFNB1, and IL6) that were unchanged in this study. These differences are likely explained by altered experimental conditions, such as differing strains of RV, culture or sampling methods including submerged culture versus air-liquid interface culture versus in vitro infection, time points of RNA sampling, microarray platforms and microarray analysis methods. Nevertheless, this well controlled study adds significant knowledge regarding the differences between healthy and COPD innate immune responses to $\mathrm{RV}-1 \mathrm{~B}$ infection under the conditions investigated, which warrant further investigation.

The underlying mechanisms of unchanged viral replication despite induced antiviral responses in COPD pBECs after RV-1B infection is currently unclear. It is possible that the IFNs produced by infection did not efficiently initiate the subsequent inductions of ISGs in COPD pBECs, therefore leading to an unchanged viral titre. However this would not explain the high apoptosis induction in COPD pBECs by RV-1B infection and marked reduction of $\mathrm{RV}-1 \mathrm{~B}$ viral replication following IFN pre-treatment. ISGs such as protein kinase R (PKR) have been shown to induce apoptosis via the Fasassociated death domain (FADD) [32], and induction of which by RV-1B infection in COPD pBECs was higher compared to that in healthy pBECs. Pre-treatment with IFNs also significantly reduced viral replication, further suggesting IFN signalling leading to ISG induction may be functional in COPD. Nevertheless, it is also possible that ISGs were ineffectively up-regulated in COPD pBECs, and apoptosis could also have been induced by other signalling pathways including TNF- $\alpha /$ TNFR1 pathway [33], and compensated for the reduced ISGs production. This may explain the excess tissue damage and high inflammation that can be caused by high levels of apoptosis in the airways of those with COPD [34].

While we showed a heightened inflammatory response and antiviral response to RV infection in COPD pBECs in this study, we also demonstrated defects in the antiviral pathway. IFN- $\beta / \lambda 1$ pre-treatment led to increased IL- 6 and TNF- $\alpha$ mRNA in healthy pBECs, however this was not translated to protein production which was either reduced or unchanged with IFN- $\beta / \lambda 1$ pre-treatment. Previous studies have shown exogenous IFN- $\beta$ decreases RV-1B-induced IL-6 release from healthy and asthmatic pBECs via an unidentified pathway [35]. IFN$\beta / \lambda 1$ pre-treatment also enhanced antiviral responses including MDA-5 and RIG-I, and IFN- $\beta$ mRNAs in healthy $\mathrm{pBECs}$, leading to marked decrease in viral titre. However, in COPD pBECs IFNs pre-treatment failed to induce MDA- 5 and RIG-I and IFN- $\beta$ mRNA, but did enhance CCL-5, CXCL-10, and IFN- $\lambda 1$ production, resulting in a decreased viral titre. This indicates that MDA-5-initiated antiviral responses were partially impaired in COPD and led to reduced IFN- $\beta$ level. It is 
possible that IFN- $\beta / \lambda 1$ pre-treatment significantly upregulated ISGs such as PKR and MxA, that bound and degraded viral RNAs as RV endocytosed into the host cells. This also suggests differential signalling pathways that regulate type III IFNs other than MDA-5/RIG-I and IFN- $\beta$. Indeed, a recent study has identified a cluster of NF- $\kappa B$ binding sites on human IFN- $\lambda 1$ promoter, and NF- $\kappa B$ was critical for IFN- $\lambda 1$ induction but not for type I IFNs expression [36]. This is consistent with our results as both NF- $\mathrm{BB}$ and IFN- $\lambda 1$ were significantly upregulated in COPD pBECs.

Apoptosis is another important component of antiviral responses, which was significantly increased in COPD pBECs after RV infection. This may correlate with the increased TNF and IFN levels [37], which can also upregulate PMAIP1 gene that promotes the induction of apoptosis [24]. However increased apoptosis did not reduce viral titre. The reason for this observation is unclear; however, it is possible that the quantification methods $\left(\mathrm{TCID}_{50}\right)$ used in this study may not be as sensitive as direct quantification methods such as plaque assays. Detection of RV-1B by qPCR could only measure the level of total viral RNAs and not differentiate live from dead viruses. Alternatively, high levels of oxidative stress could have contributed to this observation. Superoxide dismutase 2 (SOD2) and ATF3 are important antioxidative genes that were up-regulated to a greater extent in COPD pBECs after infection when compared with infected healthy pBECs. Lack of SOD2 in mice can lead to increased oxidative damage to DNA [38], and lack of ATF3 alters DNA repair mechanisms [39]. This indirectly indicates that the level of oxidative stress is higher in RV infected COPD pBECs compared to that in healthy cells.

\section{Conclusions}

We have demonstrated that COPD pBECs elicit excessive pro-inflammatory and antiviral responses to RV-1B infection. The greater expression of molecules such as the calgranulins at baseline and pellino- 1 and IRAK2 after RV-1B infection may contribute to dysregulated innate immune responses in the airways and potentiation of inflammation as seen in COPD. Additionally, MDA-5/RIG-I and IFN- $\beta$ induction to exogenous IFN- $\beta / \lambda 1$ pre-treatment prior to infection was impaired in COPD. However IFN $\lambda 1$ induction by RV-1B infection was sufficient to limit replication in COPD pBECs. This provides novel insight in the immune responses in COPD pBECs to RV infection and may reveal potential therapeutic targets that limit the dysregulated inflammatory response due to RV infection, without compromising antiviral defences in COPD.

\section{Additional file}

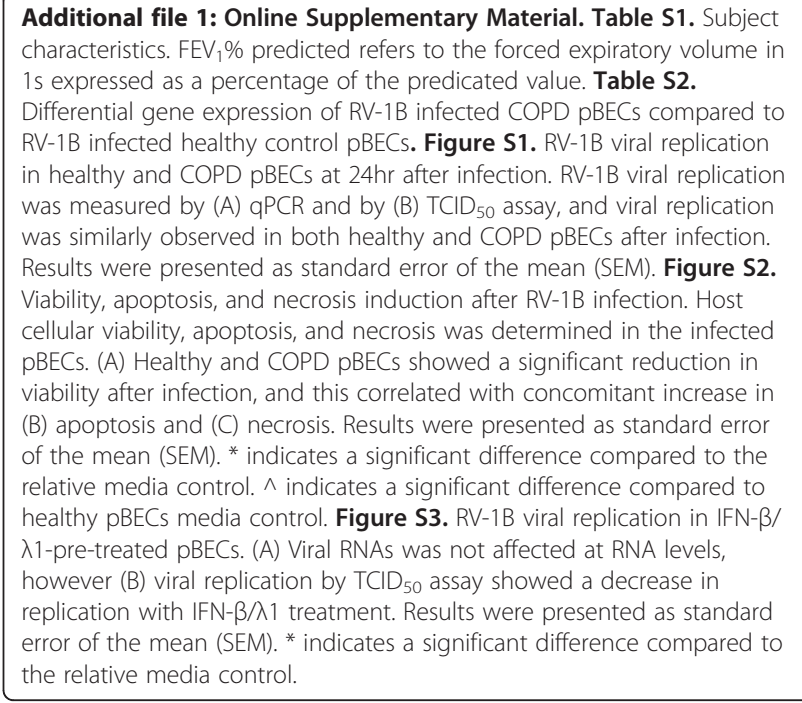

\section{Abbreviations}

RV: Rhinovirus; COPD: Chronic obstructive pulmonary disease; pBEC: Primary bronchial epithelial cell; qPCR: Quantitative real-time polymerase chain reaction; ELISA: Enzyme linked immunosorbent assay; RNA: Ribonucleic acid; IFN: Interferon; MDA-5: Melanoma differentiation-associated gene -5; RIGI: Retinoic acid-inducible gene-l; CXCL-10: Chemokine (C-X-C) motif ligand 10; CCL-5: Chemokine (C-C motif) ligand 5; TLR: Toll-like receptor; NFKB: Nuclear factor-KB; IL: Interleukin; CXCL8: Chemokine (C-X-C) motif ligand 8; FEV : Forced expiratory volume exhaled in 1 second; GOLD: Global initiative for chronic obstructive lung disease; MOI: Multiplicity of infection; Ct: Cycle threshold; TNF: Tumor necrosis factor; SEM: Standard error of means; ANOVA: Analysis of variance.

\section{Competing interests}

The authors declare they have no competing interests.

\section{Authors' contributions}

$\mathrm{KJB}, \mathrm{PGG}$, and PABW participated in the study's conception and design. PABW performed subject recruitment. KJB, MT and LPG performed the experiments. ACYH and KJB performed statistical analysis and all authors participated in the interpretation of data, preparation and editing of manuscript for intellectual content. All authors read and approved the final manuscript.

\section{Authors' information}

KJ Baines and AC-Y Hsu are joint first authors to this manuscript.

\section{Author details}

'Priority Research Centre for Asthma and Respiratory Diseases, The University of Newcastle, Callaghan, NSW, Australia. ${ }^{2}$ Virus, Infections/Immunity, Vaccines, \& Asthma, Hunter Medical Research Institute, Lot 1, Kookaburra Circuit, New Lambton Heights, NSW, Australia. ${ }^{3}$ Department of Respiratory and Sleep Medicine, John Hunter Hospital, New Lambton Heights, NSW, Australia.

Received: 2 November 2012 Accepted: 31 January 2013

Published: 6 February 2013

\section{References}

1. MacNee W: Pathogenesis of Chronic Obstructive Pulmonary Disease. Proc Am Thor Soc 2005, 2:258-266.

2. Mallia P, Message SD, Kebadze T, Parker HL, Kon OM, Johnston SL: An experimental model of rhinovirus induced chronic obstructive pulmonary disease exacerbations: a pilot study. Respir Res 2006, 7:116. 
3. Mosser AG, Brockman-Schneider R, Amineva S, Burchell L, Sedgwick JB, Busse WW, Gern JE: Similar Frequency of Rhinovirus-Infectible Cells in Upper and Lower Airway Epithelium. J Infect Dis 2002, 185:734-743.

4. Gern JE, Dick EC, Lee WM, Murray S, Meyer K, Handzel ZT, Busse WW: Rhinovirus enters but does not replicate inside monocytes and airway macrophages. J Immunol 1996, 156:621-627.

5. Seth RB, Sun L, Chen ZJ: Antiviral innate immunity pathways. Cell Res 2006, 16:141-147.

6. Chaouat A, Savale L, Chouaid C, Tu L, Sztrymf B, Canuet M, Maitre B, Housset B, Brandt C, Le Corvoisier P, Weitzenblum E, Eddahibi S, Adnot S: Role for interleukin-6 in COPD-related pulmonary hypertension. Chest 2009, 136:678-687.

7. Costa C, Rufino R, Traves SL, Lapa ESJR, Barnes PJ, Donnelly LE: CXCR3 and CCR5 chemokines in induced sputum from patients with COPD. Chest 2008, 133:26-33.

8. Hsu AC, Barr I, Hansbro PM, Wark PA: Human influenza is more effective than avian influenza at antiviral suppression in airway cells. Am J Resp Cell Mol Biol 2011, 44:906-913.

9. Baines KJ, Simpson JL, Wood LG, Scott RJ, Gibson PG: Transcriptional Phenotypes of Asthma Defined by Gene Expression Profiling of Induced Sputum Samples. J Allergy Clin Immunol 2011, 127:153-160.

10. Hsu AC, Parsons K, Barr I, Lowther S, Middleton D, Hansbro PM, Wark PAB: Critical role of type I interferon response in bronchial epithelial cell to influenza infection. PLoS One 2012, 7:e32947.

11. Cornwell WD, Kim V, Song C, Rogers TJ: Pathogenesis of Inflammation and Repair in Advanced COPD. Semin Respir Crit Care Med 2010, 31:257, 266.

12. Hogg JC, Chu F, Utokaparch S, Woods R, Elliott WM, Buzatu L, Cherniack RM, Rogers RM, Sciurba FC, Coxson HO, Pare PD: The nature of small-airway obstruction in chronic obstructive pulmonary disease. N Engl J Med 2004, 350:2645-2653.

13. Bozinovski S, Cross M, Vlahos R, Jones JE, Hsuu K, Tessier PA, Reynolds EC, Hume DA, Hamilton JA, Geczy CL, Anderson GP: S100A8 Chemotactic Protein Is Abundantly Increased, but Only a Minor Contributor to LPS-Induced, Steroid Resistant Neutrophilic Lung Inflammation in Vivo. J Proteome Res 2005, 4:136-145.

14. Castaldi PJ, Cho MH, Litonjua AA, Bakke P, Gulsvik A, Lomas DA, Anderson W, Beaty TH, Hokanson JE, Crapo JD, Laird N, Silverman EK, COPDGene ft, and Investigators E: The Association of Genome-Wide Significant Spirometric Loci with Chronic Obstructive Pulmonary Disease Susceptibility. Am J Resp Cell Mol Biol 2011, 45:1147-1153.

15. Bochkov YA, Hanson KM, Keles S, Brockman-Schneider RA, Jarjour NN, Gern JE: Rhinovirus-induced modulation of gene expression in bronchial epithelial cells from subjects with asthma. Mucosal Immunol 2010, 3:69-80.

16. Schneider D, Ganesan S, Comstock AT, Meldrum CA, Mahidhara R, Goldsmith AM, Curtis JL, Martinez FJ, Hershenson MB, Sajjan U: Increased cytokine response of rhinovirus-infected airway epithelial cells in chronic obstructive pulmonary disease. Am J Resp Crit Care Med 2010, 182:332-340.

17. Chang M, Jin W, Sun SC: Peli1 facilitates TRIF-dependent Toll-like receptor signaling and proinflammatory cytokine production. Nat Immunol 2009, 10:1089-1095.

18. Keating SE, Maloney GM, Moran EM, Bowie AG: IRAK-2 participates in multiple toll-like receptor signaling pathways to NFkappaB via activation of TRAF-6 ubiquitination. J Biol Chem 2007, 282:33435-33443.

19. Diczfalusy U, Olofsson KE, Carlsson A-M, Gong M, Golenbock DT, Rooyackers O, FIÃring U, BjÃrkbacka H: Marked upregulation of cholesterol 25-hydroxylase expression by lipopolysaccharide. J Lipid Res 2009, 50:2258-2264

20. Bennett JA, Prince LR, Parker LC, Stokes CA, De Bruin HG, Van Dern Berge M, Heijink IH, Whyte MK, Sabroe I: Pellino-1 Selectively Regulates Epithelial Cell Responses to Rhinovirus. J Virol 2012, epub ahead of print 18 April.

21. Kawagoe T, Sato S, Matusushita K, Kato H, Matsui K, Kumagai Y, Saitoh T, Kawai T, Takeuchi O, Akira S: Sequential control of Toll-like receptordependent responses by IRAK1 and IRAK2. Nat Immunol 2008, 9:684-691.

22. Jin W, Chang M, Sun SC: Peli: a family of signal-responsive E3 ubiquitin ligases mediating TLR signaling and T-cell tolerance. Cell Mol Immunol 2012, 9:113-122.

23. Smith PM, Jacque B, Conner JR, Poltorak A, Stadecker MJ: IRAK-2 Regulates IL-1-Mediated Pathogenic Th17 Cell Development in Helminthic Infection. PloS Pathog 2011, 7:e1002272.
24. Sun Y, Leaman DW: Involvement of Noxa in Cellular Apoptotic Responses to Interferon, Double-stranded RNA, and Virus Infection. J Biol Chem 2005, 280:15561-15568.

25. Chen BP, Wolfgang CD, Hai T: Analysis of ATF3, a transcription factor induced by physiological stresses and modulated by gadd153/Chop10. Mol Cell Biol 1996, 16:1157-1168.

26. Suganami T, Yuan X, Shimoda Y, Uchio-Yamada K, Nakagawa N, Shirakawa I, Usami T, Tsukahara T, Nakayama K, Miyamoto Y, Yasuda K, Matsuda J, Kamei Y, Kitajima S, Ogawa Y: Activating Transcription Factor 3 Constitutes a Negative Feedback Mechanism That Attenuates Saturated Fatty Acid/ Toll-Like Receptor 4 Signaling and Macrophage Activation in Obese Adipose Tissue. Circ Res 2009, 105:25-32.

27. Hu Y, Wang J, Yang B, Zheng N, Qin M, Ji Y, Lin G, Tian L, Wu X, Wu L, Sun $B$ : Guanylate binding protein 4 negatively regulates virus-induced type I IFN and antiviral response by targeting IFN regulatory factor 7 . J Immunol 2011, 187:6456-6462.

28. Bazan JF, Bacon KB, Hardiman G, Wang W, Soo K, Rossi D, Greaves DR, Zlotnik A, Schall TJ: A new class of membrane-bound chemokine with a CX3C motif. Nature 1997, 385:640-644.

29. Schneider D, Ganesan S, Comstock AT, Meldrum CA, Mahidhara R, Goldsmith AM, Curtis JL, Martinez FJ, Hershenson MB, Sajjan U: Increased cytokine response of rhinovirus-infected airway epithelial cells in chronic obstructive pulmonary disease. Am J Respir Crit Care Med 2010, 182:332-340.

30. Proud D, Turner RB, Winther B, Wiehler S, Tiesman JP, Reichling TD, Juhlin KD, Fulmer AW, Ho BY, Walanski AA, Poore CL, Mizoguchi H, Jump L, Moore ML, Zukowski CK, Clymer JW: Gene Expression Profiles during In Vivo Human Rhinovirus Infection: Insights into the Host Response. Am J Respir Crit Care Med 2008, 178:962-968.

31. Chen Y, Hamati E, Lee P-K, Lee W-M, Wachi S, Schnurr D, Yagi S, Dolganov G, Boushey H, Avila P, Wu R: Rhinovirus Induces Airway Epithelial Gene Expression through Double-Stranded RNA and IFN-Dependent Pathways. American Journal of Respiratory Cell and Molecular Biology 2006, 34:192-203.

32. Gil J, Esteban M: The interferon-induced protein kinase (PKR), triggers apoptosis through FADD-mediated activation of caspase 8 in a manner independent of Fas and TNF-alpha receptors. Oncogene 2000, 19:3665-3674

33. Micheau O, Tschopp J: Induction of TNF receptor I-mediated apoptosis via two sequential signaling complexes. Cell 2003, 114:181-190.

34. Hodge S, Hodge G, Holmes M, Reynolds PN: Increased airway epithelial and T-cell apoptosis in COPD remains despite smoking cessation. Eur Respir J 2005, 25:447-454.

35. Cakebread JA, Xu Y, Grainge C, Kehagia V, Howarth PH, Holgate ST, Davies DE: Exogenous IFN-beta has antiviral and anti-inflammatory properties in primary bronchial epithelial cells from asthmatic subjects exposed to rhinovirus. J Allergy Clin immunol 2011, 127:1148-1154. e9.

36. Thomson SJ, Goh FG, Banks H, Krausgruber T, Kotenko SV, Foxwell BM, Udalova IA: The role of transposable elements in the regulation of IFN-lambda1 gene expression. Proc Natl Acad Sci U S A 2009, 106:11564-11569.

37. Chawla-Sarkar M, Lindner DJ, Liu YF, Williams BR, Sen GC, Silverman RH, Borden EC: Apoptosis and interferons: role of interferon-stimulated genes as mediators of apoptosis. Apoptosis 2003, 8:237-249.

38. Van Remmen H, Ikeno Y, Hamilton M, Pahlavani M, Wolf N, Thorpe SR, Alderson NL, Baynes JW, Epstein CJ, Huang TT, Nelson J, Strong R, Richardson A: Life-long reduction in MnSOD activity results in increased DNA damage and higher incidence of cancer but does not accelerate aging. Physiol Genomics 2003, 16:29-37.

39. Turchi L, Fareh M, Aberdam E, Kitajima S, Simpson F, Wicking C, Aberdam D, Virolle T: ATF3 and p15PAF are novel gatekeepers of genomic integrity upon UV stress. Cell Death Differ 2009, 16:728-737.

\section{doi:10.1186/1465-9921-14-15}

Cite this article as: Baines et al:: Novel immune genes associated with excessive inflammatory and antiviral responses to rhinovirus in COPD. Respiratory Research 2013 14:15. 